Article

\title{
Blood Pressure Estimation Algorithm Based on Photoplethysmography Pulse Analyses
}

\author{
Seon-Chil Kim ${ }^{1}\left(\mathbb{D}\right.$ and Sung-Hyoun Cho ${ }^{2, *}$ (D) \\ 1 Department of Biomedical Engineering, School of Medicine, Keimyung University, 1095 Dalgubeol-daero, \\ Daegu 42601, Korea; chil@kmu.ac.kr \\ 2 Department of Physical Therapy, Nambu University, 23 Cheomdan Jungang-ro, Gwangsan-gu, \\ Gwangju 62271, Korea \\ * Correspondence: shcho@nambu.ac.kr; Tel.: +82-10-3060-1330; Fax: 82-62-970-0492
}

Received: 15 May 2020; Accepted: 8 June 2020; Published: 12 June 2020

\begin{abstract}
Though small wrist-type blood pressure (BP) measurement devices using simple sensors have recently been developed, they lack accuracy and require the input of personal user data. To miniaturize BP measurement devices, this study proposes the use of algorithms that can calculate BP using sensors that utilize only two photoplethysmographies (PPGs), without the need for electrocardiograms. To reduce the error of indirect BP measurement, specific values representing individual blood vessel conditions are improved via the application of an algorithm developed using mean arterial pressure and PPG phase difference. To reduce the error that occurs when measuring BP using PPG, a specific value algorithm used to develop a miniaturized BP measuring device using two PPGs is proposed along with a new model. The developed ultra-small PPG measuring device uses a reflective photoplethysmography measurement circuit. This is presented as a PPG-only model that deviates from the method of additionally linking existing electrocardiography (ECG). The measurement device developed in this study is compared with the air-pressurized automatic BP measurement device in terms of the BP measurement value; the result of maintaining the error stably at $5 \%$ is derived to prove the superiority of the algorithm.
\end{abstract}

Keywords: blood pressure; photoplethysmography; systolic blood pressure; diastolic blood pressure; pulse interval; mean arterial pressure

\section{Introduction}

The blood that flows through the body contains a great deal of information. In particular, pulse velocity can be used as an indicator of arterial stiffness and health [1-3]. Specifically, blood pressure (BP) information is used as an important indicator of health management for patients with chronic diseases such as hypertension and arteriosclerosis. The most preferred method used to measure BP is based on pressure-cuff measurements, whereby the vessel is collapsed following (a) the application of external pressure, and (b) its subsequent release and auditory sound auscultation. Most clinicians recognize air pressurization BP measurements to represent credible BP values [4-6]. However, this technique involves an upper-arm air pressurization methodology that in turn involves a complicated inspection process, which is often difficult for users to perform on their own. Additionally, it lacks the capacity to perform real-time measurements. Therefore, the demand for ultra-small BP measuring devices has recently been increasing. Furthermore, owing to the burgeoning popularity of wearable healthcare devices, many small wearable wrist-type BP measuring instruments have been developed $[7,8]$.

Most wearable blood pressure measuring devices differ from those of the cuff method, which determines the absolute blood pressure value and is used by medical institutions. 
Accordingly, the final measurements vary depending on the area of the measurement and the physical conditions. Specifically, measurement methods employed by existing wearable BP measurement devices involve the indirect inference of BP via the calculation of characteristics and correlations such as blood flow velocity and time [9].

The pulse wave velocity (PWV) and pulse transit time (PTT) are affected by the distance from the heart to the measuring sensor, which in turn affects blood pressure [10,11]. However, this indirect measurement method faces challenges in terms of using the information related to personal health conditions that affect PWV and PTT, such as the size of the vessel (representing the vessel's condition), blood concentration, and elasticity of the vessel wall [12]. Many existing studies have therefore proposed algorithms based on the correlation between PTT and photoplethysmography (PPG) in order to directly measure the factors that influence BP $[13,14]$. It is known that the weight, mass of body fat, percentage of body fat, arm circumference, arm length, and height of an individual are the physical factors that directly affect PTT [15].

$\mathrm{BP}$ can be calculated by measuring blood flow from the heart to the PPG measurement sensor in the peripheral blood vessel. The PPG measurement sensor can infer BP by measuring the pulse wave in peripheral blood vessels. If the variability of the electrocardiography (ECG) waves can be coupled to individual physical data via algorithms, the inconvenience of entering user-specific information can hypothetically be minimized or eliminated via the transformation of PPG waves into non-linear data whenever BP is measured using a PPG sensor. In this study, we develop a method to deduce the variations in phase difference, blood vessel size, blood concentration, and elasticity of blood vessels among various individuals using only two PPGs based on the correlation between the average arterial pressure and BP.

In order to reduce the error of the existing PPG estimated BP device, it is necessary to calculate and input the blood vessel cross-sectional area, cardiac output, and blood specific gravity, which are specific values that represent the condition of an individual's blood vessels. However, in this study, errors in BP measurement values can be minimized by utilizing only the phase difference between mean arterial pressure (MAP) and PPG.

\section{Materials and Methods}

Systolic blood pressure (SBP) and diastolic blood pressure (DBP) information can be obtained as per existing studies using PTT from PPG data measured noninvasively at the fingers [16]. The BP and PTT are inversely correlated. Specifically, small PTT values correspond to short blood transfer times and highly rigid blood vessels, demonstrating that blood flow rates in blood vessels are high [17]. Therefore, we propose that the individual's vascular conditions could affect the PPG. A graph representing the measured finger PPG is shown in Figure 1, and was obtained by filtering two single wavelengths using an infrared light-emitting diode of $940 \mathrm{~nm}$. In this study, PPGs were collected and analyzed (Figure 1). The peak starting point of the PPGs can be identified using ECGs. Recognizing the initial ECG R peak of the PPG as R1 allows for continuous BP estimation with two PPGs without any additional ECG measurements [18,19].

Therefore, PTT can be defined as the time taken by the pulse wave generated by the heart at R1 to reach P1 of the 1st PPG. The pulse interval time (PT) of P1 and P2 can then be quantified on the basis of the differences between the two PPGs. According to the Bramwell-Hill model, the PWV can be defined [20] as

$$
\mathrm{PWV}=\frac{L}{P T}=\sqrt{\frac{V_{d i a} \Delta P}{\rho \Delta V}}
$$

where the distance between the sensors is denoted by $L$, and the time difference between the two PPGs is denoted by $P T$. 


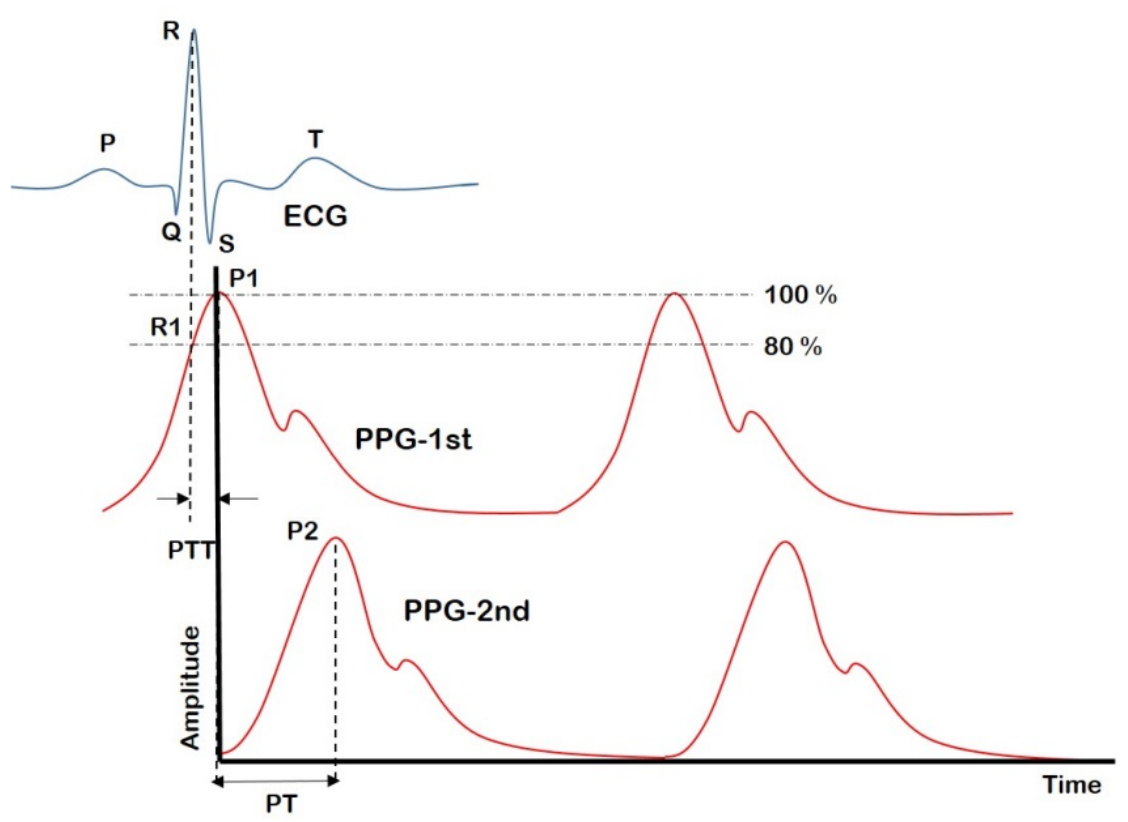

Figure 1. Analyzed results based on recordings from two photoplethysmographies (PPGs). PTT: pulse transit time, PT: the time difference between the two PPGs P1 and P2.

In the above equation, $V$ is the cross-sectional area of a blood vessel, and $\rho$ is its specific gravity. Moreover, $\Delta \mathrm{P}$ is the difference between the pulse pressure and $\mathrm{DBP}$, and $\Delta \mathrm{V}=\mathrm{V}_{\text {dia }}-\mathrm{V}_{\text {sys }}$ [18]. Furthermore, $\rho$ varies in different individuals and is difficult to estimate using a PPG. If the specific values of individual $\mathrm{V}$ and $\rho$ are set as constant values, and the distance is fixed, the following Equation (2) can be obtained:

$$
\mathrm{P}-\mathrm{DBP}=\frac{\Delta V \cdot \rho L^{2}}{V_{d i a} \cdot P T^{2}}=\frac{\alpha}{P T^{2}}
$$

Furthermore, BP can be estimated using the mean arterial pressure (MAP). In turn, MAP can be estimated as the sum of the central venous pressure to the product of the cardiac output (CO) and system vascular resistance (SVR) [21]. The central venous pressure is very small and can be ignored. Additionally, the cardiac output is defined as the product of the heart rate (HR) and stroke volume (SV). Thus, MAP can be defined as follows [22]:

$$
\text { MAP }=\text { HR.SV·SVR }
$$

It can be alternatively defined as $\mathrm{MAP}=(\mathrm{SBP}+2 \mathrm{DBP}) / 3[23]$ and can be expressed as:

$$
\mathrm{SBP}+2 \mathrm{DBP}=3(\mathrm{HR} \cdot \mathrm{SV} \cdot \mathrm{SVR})
$$

SV and SVR are individual values and cannot be obtained from the PPG measurement; therefore, they are summarized as the constant. The heart rate (HR) is the same as the peak number of the pulse rate (PR) in the PPG measurement [24].

Therefore, DBP and SBP can be obtained by calculating the parameters shown in Equation (5) using two PPG sensors on the basis of the Bramwell-Hill model,

$$
\mathrm{DBP}=\left(P R \cdot \alpha-\frac{1}{3}\right)\left(\frac{b}{P T^{2}}\right), \mathrm{SBP}=\left(P R \cdot \alpha+\frac{2}{3}\right)\left(\frac{b}{P T^{2}}\right)
$$

where PT is the interval between P1 and P2. 
In order to calculate the BP value accurately using the PPG, a and b, treated as constants, can be calculated as in Equation (6) [18]. Equation (6) can be obtained from Equations (4) and (5).

$$
\alpha=\mathrm{MAP} /(\mathrm{PP} \cdot \mathrm{PR}), \mathrm{b}=\mathrm{PP} \cdot P T^{2}
$$

To estimate the BP values, the input SBP and DBP from existing BP values can be obtained, whereas the remaining values can be obtained via algorithms. Because PR and PT can be obtained using PPGs, specific values of $a$ and $b$ for different individuals can be obtained using individual PPGs.

A device capable of measuring PPG consists of a sensor module and an amplification, conversion, and signal transmission unit. The sensor, which is a measuring unit, consists of a red-light-wave measuring circuit for reflection-type light, using infrared light-emitting and light-receiving diodes. More than 20 PPG waveforms were sampled through two channels and measured in a constant area through filtering. The sampling reduced the time for repeated measurements by measuring in a narrow band around $64 \mathrm{~Hz}$ to reduce noise, and PTT set the peak value through differential filtering. In addition, to eliminate distortion due to noise around the measurement area, areas of $0.5 \mu \mathrm{s}$ or less and $10 \mu$ s or more were filtered, and variable amplification signal bands $(0.5 \mathrm{~Hz}$ to $5 \mathrm{~Hz})$ were designated and processed.

This study was approved by the institutional review board (IRB) of Nambu University, Gwangju city, South Korea (NBU-IRB-1041478-2017-HR-017) and conducted in accordance with the ethical standards of the declaration of Helsinki. All subjects were informed of the purpose of the study and study design, and all participants provided written informed consent.

To verify the BP estimation algorithm, SBP and DBP were obtained through the automatic sphygmomanometer of the cuff method and PPG measurement device developed in this study. The experimental data were based on 8 men and 13 women (average age: 21.10 years, standard deviation: 1.64 years), who did not have circulatory diseases, which were obtained using the automatic BP cuff measurement method and PPG. After resting for 10 minutes before the test, the average values for SBP and DBP were obtained from both devices by measuring twice over a period of 10 days in the same posture, as shown in Figure 2. The automatic blood pressure monitor (ACCUNI, BP210, Seoul, South Korea) was used as a gold standard, as it is recognized as an absolute value of blood pressure measurement by medical institutions. It is a device that satisfies the standards of the American National Standards Institute (ANSI)/Association for the Advancement of Medical Instrumentation (AAMI) and was used for measurements in the same environment as PPG [25].

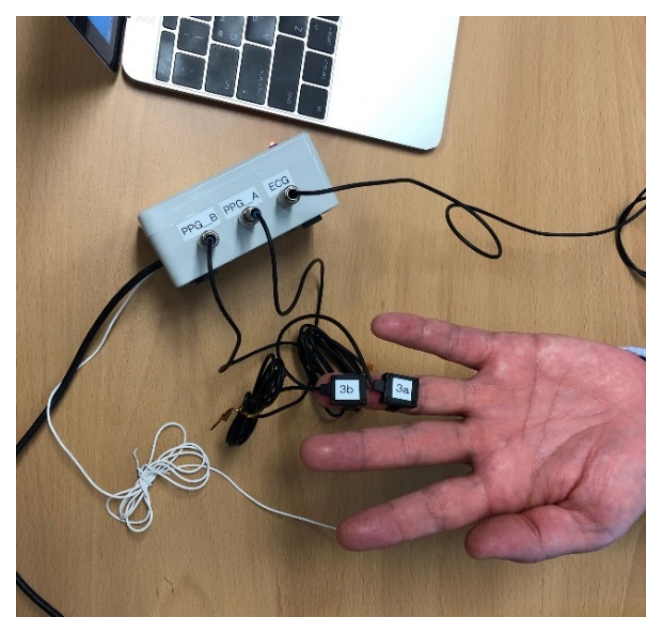

(a)

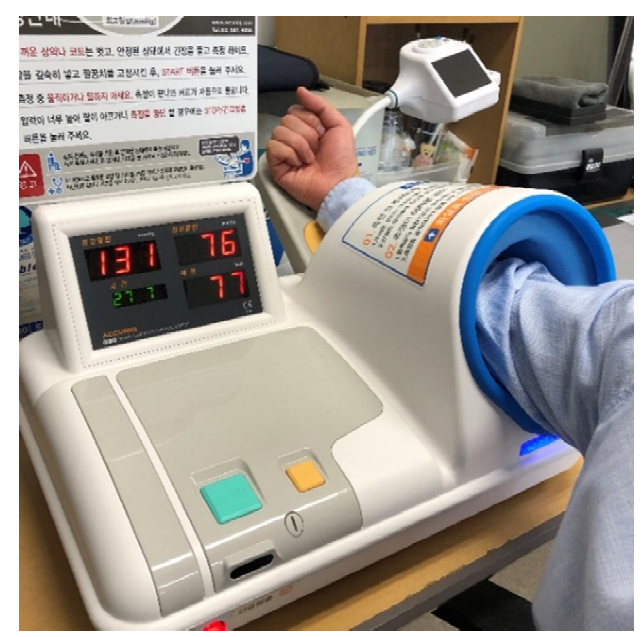

(b)

Figure 2. Equipment used for blood pressure measurements. (a) PPG measurement method, (b) Automatic blood pressure measurement method via air pressurization. 


\section{Results}

The measurement screen, as shown in Figure 3, was obtained through PPG filtering. Figure 3a shows the results of the ECG and PPG measurements in time, confirming that the peak of the R wave and the intersection of the PPG were 80\%, as shown in Figure 1, to obtain the PTT. Therefore, as shown in Figure 3b, it was possible to obtain both values necessary for BP measurement via two PPG measurements. Therefore, the sampling was repeated, and the values automatically measured. As shown in Figure $3 b$, a program was implemented for automatic measurements with repetitions.

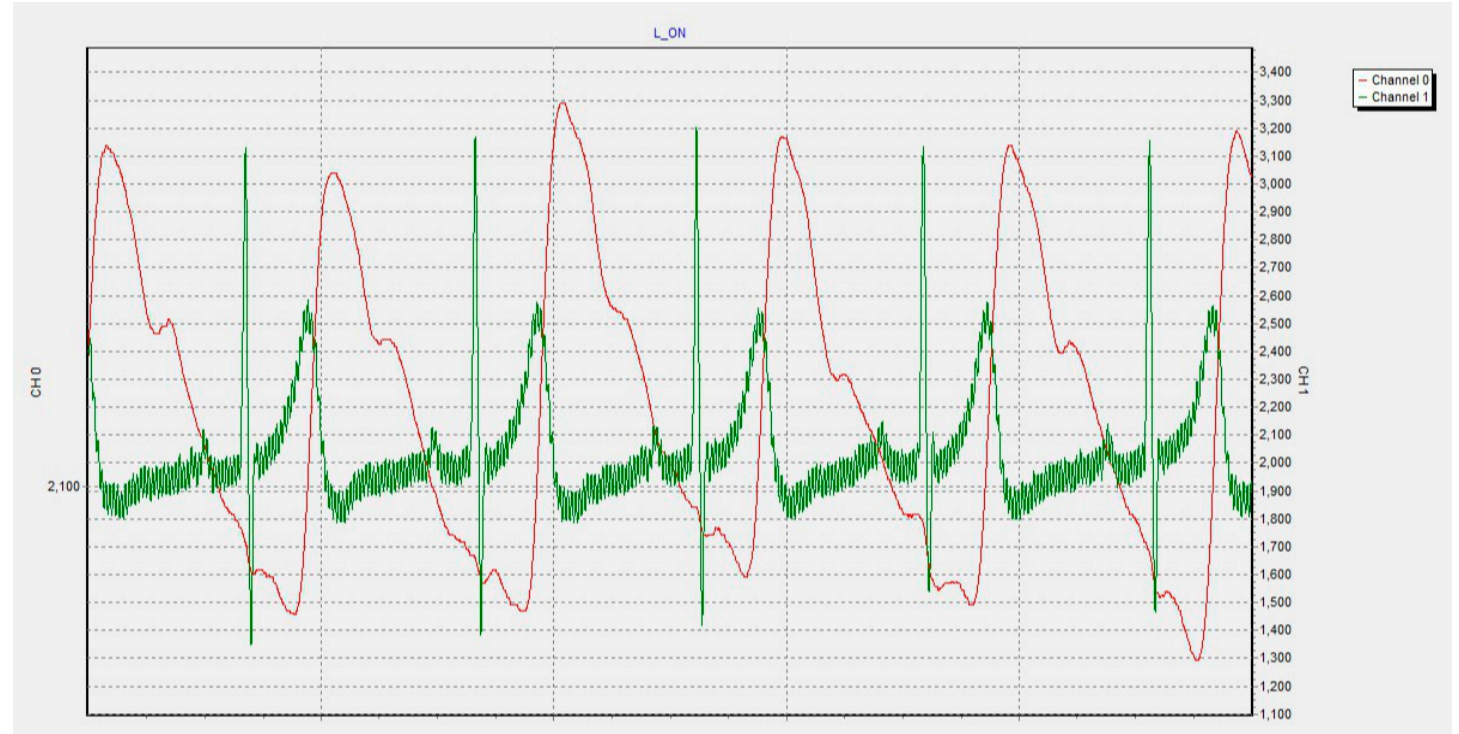

(a)

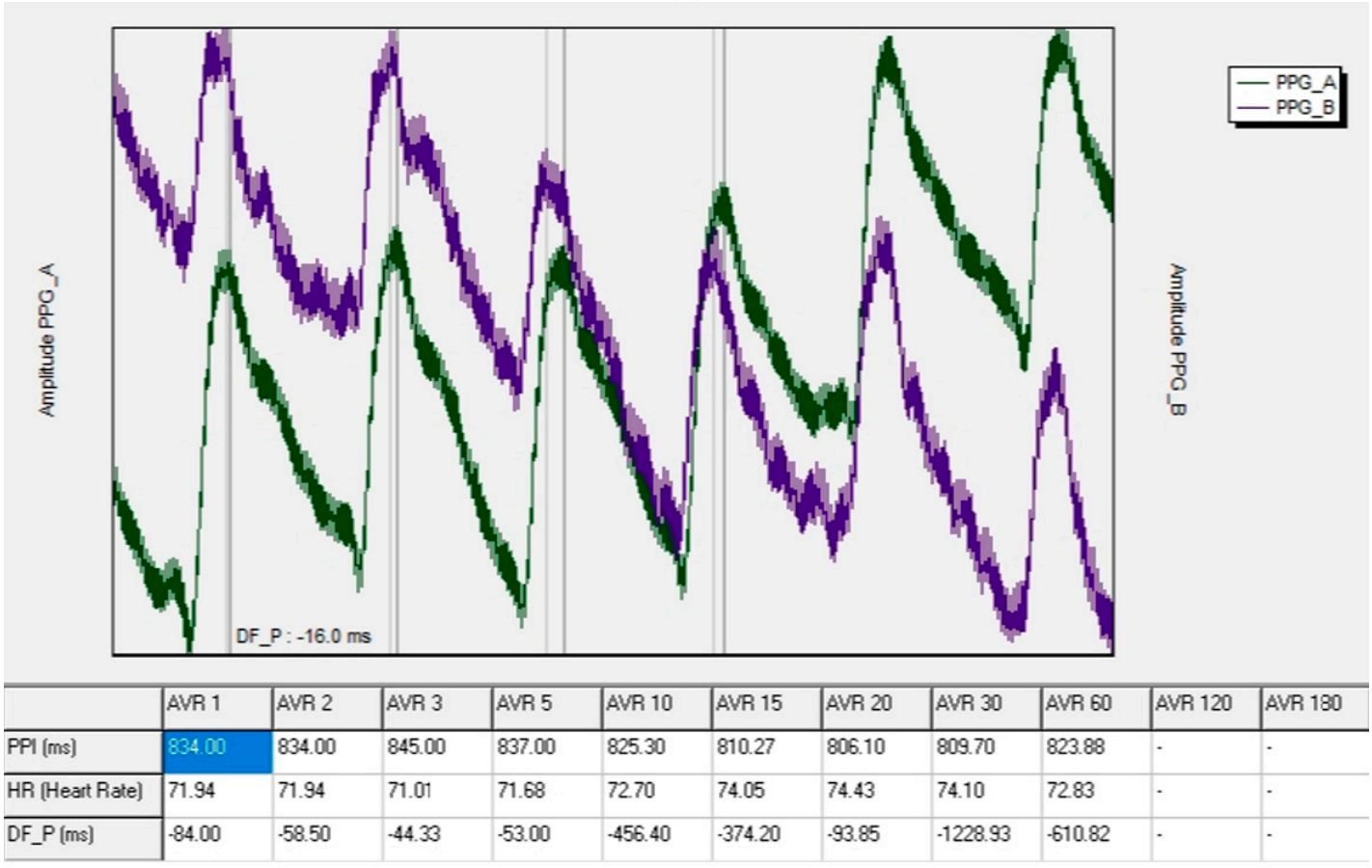

(b)

Figure 3. Images showing PPG measurements plotted on the monitor of the device. (a) Measurement by changing the PTT standard through electrocardiography (ECG) and PPG, (b) Measurement of PT, PTT, and pulse rate (PR) through two PPGs. 
Using the two PPGs measurements obtained from the fingers, the MAP algorithm presented in this study yielded the specific values for constants $a$ and $b$ for different individuals (Table 1 ). These values could be obtained in the same manner by which the values were obtained in the case of SBP and DBP. The correlations between SBP and DBP, and PT and MAP can be analyzed to study the linear relationships between them (Figure 4).

Table 1. Estimated constants $a$ and $b$ based on the use of PPG $(\mathrm{N}=21)$.

\begin{tabular}{|c|c|c|c|c|c|c|c|c|}
\hline \multirow{2}{*}{ Variable } & \multicolumn{3}{|c|}{ Electronic BP Measurement (mmHg) } & \multicolumn{3}{|c|}{ PPG Estimation Algorithm } & \multirow{2}{*}{ a } & \multirow{2}{*}{$\mathbf{b}$} \\
\hline & SBP & DBP & PP & PT & PR & MAP & & \\
\hline Mean & 115.61 & 75.00 & 40.60 & 36.71 & 67.19 & 88.48 & 0.032811 & $55,186.8$ \\
\hline $\begin{array}{l}\text { Standard } \\
\text { deviation }\end{array}$ & 5.20 & 5.18 & 3.65 & 2.27 & 2.82 & 4.89 & 0.003985 & $11,027.23$ \\
\hline
\end{tabular}

BP: blood pressure; PPG: photoplethysmography; SBP: systolic blood pressure; DBP: diastolic blood pressure; MAP: mean arterial pressure; PT: interval between P1 and P2; PP: SBP - DBP.

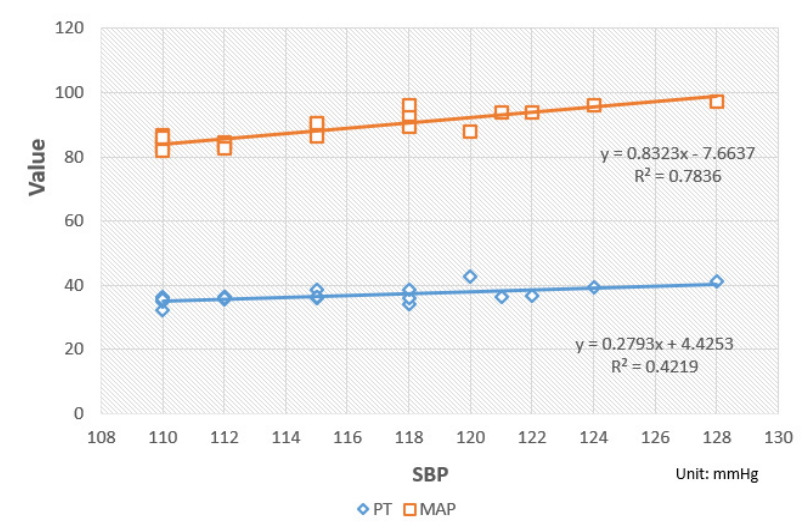

(a)

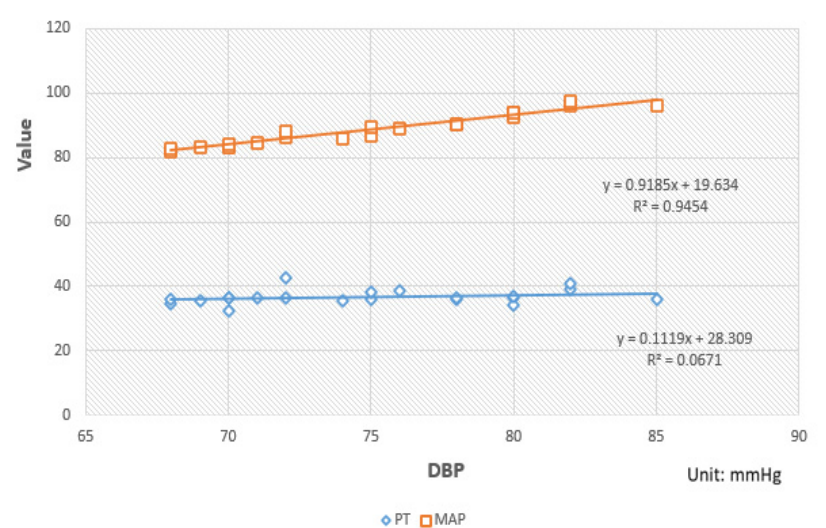

(b)

Figure 4. Systolic blood pressure (SBP), diastolic blood pressure (DBP), mean arterial pressure (MAP), and PT correlation analyses. (a) Analysis of the correlation of SBP with PT and MAP; (b) Analysis of the correlation of DBP with PT and MAP.

The higher the SBP, the more the PT appeared, and the more the MAP tended to increase. PTT appeared to decrease as the SBP increased, which was consistent with the tendency of small-time difference between the two PPG peaks. Upon comparison of the graph with Figure 4, it was observed that SBP tended to be more affected by MAP than was the DBP. It can therefore be said that an individual-specific value of blood vessels tends to be affected by SBP. The MAP had the same effect on SBP and DBP, but the $\mathrm{R}^{2}$ value for DBP was as low as 0.0671, while that for SBP was as high as 0.4219. 
The specific values of constants $a$ and $b$ were estimated for specific individuals for the same target. Then, the error (\%) of the estimated BP, calculated via the algorithm using PPG for 10 days, and the absolute value, measured using the automatic BP device, were estimated, as listed in Table 2. The SBP yielded a slightly lower error rate than the DBP, and the value measured using the PPG yielded a stable error value of approximately $5 \%$.

Table 2. Difference between estimated and measured blood pressure mean values $(\mathrm{N}=21)$.

\begin{tabular}{|c|c|c|c|c|c|c|}
\hline \multirow[t]{2}{*}{ Variable } & \multicolumn{2}{|c|}{$\begin{array}{l}\text { PPG Estimation } \\
\text { Algorithm } \\
\text { BP (mmHg) }\end{array}$} & \multicolumn{2}{|c|}{$\begin{array}{c}\text { Electronic Blood } \\
\text { Pressure Measurement } \\
\text { BP (mmHg) }\end{array}$} & \multirow{2}{*}{$\begin{array}{l}\text { SBP } \\
\text { Error } \\
(\%)\end{array}$} & \multirow{2}{*}{$\begin{array}{c}\text { DBP } \\
\text { Error } \\
(\%)\end{array}$} \\
\hline & SBP & DBP & SBP & DBP & & \\
\hline Mean & 118.91 & 79.40 & 115.01 & 75.00 & 3.391 & 5.866 \\
\hline $\begin{array}{l}\text { Standard } \\
\text { deviation }\end{array}$ & 5.23 & 5.16 & 5.17 & 5.26 & 0.867 & 2.273 \\
\hline
\end{tabular}

SBP (DBP) Error (\%) = (PPG Estimation Algorithm BP - Electronic Blood Pressure Measurement BP)/Electronic Blood Pressure Measurement BP $\times 100$.

\section{Discussion}

Medical institutions measure BP using the air pressure measurement method. Many healthcare products have recently been developed for the measurement of $\mathrm{BP}$, but the data obtained using PPG sensors are inaccurate compared with those obtained via the air pressurization method. Thus, the measurement values and the validity of the algorithm are not reliable. The largest errors in these measurements obtained when PPG sensors are used are attributed to the estimation of specific biomarker values [26].

When BP is measured using the PPG, BP values close to those obtained via the air pressure method can be obtained only by inputting body information such as the individual's height and weight, or by entering a blood vessel's cross-sectional area, cardiac output, etc. [27]. However, it is cumbersome to enter these values prior to PPG measurement. The PPG measurement method is an essential condition because it facilitates the reduction of errors in the measured value by utilizing the prior history of the individual-specific value [28].

Photoplethysmography is a measurement method used to analyze blood flow in the blood vessel. It employs PPGs that measure or estimate various biomarkers, such as PWV and PTT, that are highly useful for BP measurements $[29,30]$. Among the factors that directly reflect individual vascular conditions, this study emphasizes MAP and helps to utilize the information about the blood transfer by the heart and its relationship with blood vessels.

It was hypothesized, as mentioned in Section 1, that if a method were used to obtain specific values to analyze the conditions of blood vessels of an individual based on the absolute value of a specific biomarker pertaining to the blood vessels, this would constitute an alternative method to reduce the errors of the measured values using PPG, and would thereby allow for successful implementation of the algorithm. In this process, the cross-sectional area of the blood vessel (V), specific gravity of blood $(\rho)$, SVR, and SV were defined as specific values that could express the condition of the blood vessels [31]. We implemented an algorithm that could deduce biomarker values based on the use of the MAP.

Algorithms developed in previous studies used the Moens-Korteweg model and the PWV. However, these used the same input values, such as the elasticity of blood vessels, vascular diameter, and vascular thickness as constants [32]. To improve BP measurement accuracy in this study, $\mathrm{BP}$ was obtained using an equation that combined specific values from individuals with real-time PPG measurements.

This equation yielded BP measurements with the use of the MAP presented in this study with an error rate of $5 \%$, as proposed by the SBP and DBP estimation algorithms. In addition, certain values 
that can determine an individual's blood vessel status can be easily inferred from the initial BP values of the individual. This can be considered a new model for BP measurements with the use of PPG. This study developed an approach that can measure the changes in specific values of specific age groups to study and establish criteria for different vascular conditions. We believe that this method can be developed into a new health measurement system because it can estimate the health status of blood vessels using BP values. However, the applicability of the findings of this study is limited because the validity of the developed algorithm was only based on healthy young people (in their 20s) without circulatory system illnesses. This study originated with the idea of measuring BP by inserting a PPG sensor on the back of a clock such as an Apple Watch. Most of the errors in previous studies were caused by physical conditions or the health condition of the blood vessels. ECGs are being used to improve accuracy, but there is some hassle in measuring them. Therefore, this study proposes a device that measures with only two PPGs and extracts unique values, such as a person's physical condition, from existing BP values to increase the accuracy, as shown in the results of the experiment. The gold-standard device for blood pressure values (the cuff-type automatic blood pressure device) had some deviation in BP values as compared to those measured by the PPG device.

The BP estimation algorithm presented in this study can also be applied to the miniaturization of BP measurement devices and for performing continuous, real-time BP measurements. Furthermore, the developed model is expected to be able to aid in the management of health conditions of patients with chronic conditions and reduce medical expenses because it generates safer and more reliable measurements compared with those of existing devices that use PPG sensors.

\section{Conclusions}

In this study, a new algorithm was proposed to analyze the specific values of individuals with vascular conditions. Specific values related to the individual who was tested were inputted into the BP estimation algorithm, and these were processed using the PPG phase difference, time difference, and MAP measurements. These values contributed to the improvement of the accuracy of the BP estimation value via the PPG sensor.

Specifically, MAP affected parameters such as SBP, DBP, and PT. Furthermore, the phase difference between the two PPGs played an important role in BP estimation. Moreover, BP measurements based on the developed algorithm yielded a stable error rate of $\sim 5 \%$ compared with that of the existing air pressurization measurement method, thereby demonstrating the validity of the algorithm. The proposed algorithm can be applied to the miniaturization of BP measurement devices and to continuous real-time BP measurements.

Author Contributions: Project administration, S.-C.K. and S.-H.C.; methodology, S.-H.C.; software, S.-C.K.; investigation, S.-C.K.; writing—original draft preparation, S.-C.K.; writing—review and editing, S.-H.C. All authors have read and agreed to the published version of the manuscript.

Funding: This research received no external funding.

Acknowledgments: We would like to thank Editage (www.editage.co.kr) for English language editing.

Conflicts of Interest: The authors declare no conflicts of interest.

\section{References}

1. Cruickshank, K.; Riste, L.; Anderson, S.G.; Wright, J.S.; Dunn, G.; Gosling, R.G. Mb Aortic Pulse-Wave Velocity and Its Relationship to Mortality in Diabetes and Glucose Intolerance. Circulation 2002, 106, 2085-2090. [CrossRef] [PubMed]

2. Sun, K.; Daimon, M.; Watanabe, S.; Komuro, I.; Masuda, Y. The relation of pulse wave velocity by oscil-lometric and tonometric methods and clinical application studies. Jpn. J. Appl. Physiol. 2002, 32, 81-86.

3. Pilt, K.; Meigas, K.; Kööts, K.; Viigimaa, M. Photoplethysmographic signal rising front analysis for the discrimination of subjects with increased arterial ageing. Proc. Estonian Acad. Sci. 2014, 63, 221. [CrossRef] 
4. Wu, W.; Lee, J.; Chen, H. Estimation of Heart Rate Variability Changes during Different Visual Stimulations Using Non-invasive Continuous ECG Monitoring System. In Proceedings of the 2009 International Joint Conference on Bioinformatics, Systems Biology and Intelligent Computing, Shanghai, China, 3-6 August 2009; pp. 344-347.

5. Staessen, J.A.; O'Brien, E.T.; Thijs, L.; Fagard, R.H. Modern approaches to blood pressure measurement. Occup. Environ. Med. 2000, 57, 510-520. [CrossRef] [PubMed]

6. O’Brien, E.; Pickering, T.; Asmar, R.; Myers, M.; Parati, G.; Staessen, J.A.; Mengden, T.; Imai, Y.; Waeber, B.; Palatini, P.; et al. Working Group on Blood Pressure Monitoring of the European Society of Hypertension International Protocol for validation of blood pressure measuring devices in adults. Blood Press. Monit. 2002, 7, 3-17. [CrossRef]

7. Asada, H.H.; Shaltis, P.; Reisner, A.; Rhee, S.; Hutchinson, R.C. Mobile monitoring with wearable photoplethysmographic biosensors. IEEE Eng. Med. Boil. Mag. 2003, 22, 28-40. [CrossRef]

8. Salvi, P.; Lio, G.; Labat, C.; Ricci, E.; Pannier, B.; Benetos, A. Validation of a new non-invasive portable tonometer for determining arterial pressure wave and pulse wave velocity. J. Hypertens. 2004, 22, $2285-2293$. [CrossRef] [PubMed]

9. Kim, J.; Kim, I.G. Flexible and Wearable Pressure Sensor for Biomedical Applications. J. Korean Inst. Commun. Inf. Sci. 2018, 43, 1694-1702. [CrossRef]

10. Sharifi, I.; Goudarzi, S.; Khodabakhshi, M.B. A novel dynamical approach in continuous cuffless blood pressure estimation based on ECG and PPG signals. Artif. Intell. Med. 2019, 97, 143-151. [CrossRef] [PubMed]

11. Lazazzera, R.; Belhaj, Y.; Carrault, G. A new wearable device for blood pressure estimation using pho-toplethysmogram. Sensors 2019, 19, 2557. [CrossRef]

12. Wang, L.; Zhou, W.; Xing, Y.; Zhou, X. A Novel Neural Network Model for Blood Pressure Estimation Using Photoplethesmography without Electrocardiogram. J. Heal. Eng. 2018, 2018, 1-9. [CrossRef] [PubMed]

13. Liu, M.; Po, L.-M.; Fu, H. Department of Computer Science, Chu Hai College of Higher Education, Hong Kong SAR, China Cuffless Blood Pressure Estimation Based on Photoplethysmography Signal and Its Second Derivative. Int. J. Comput. Theory Eng. 2017, 9, 202-206. [CrossRef]

14. Perpetuini, D.; Chiarelli, A.M.; Cardone, D.; Rinella, S.; Massimino, S.; Bianco, F.; Bucciarelli, V.; Vinciguerra, V.; Fallica, P.; Perciavalle, V.; et al. Photoplethysmographic Prediction of the Ankle-Brachial Pressure Index through a Machine Learning Approach. Appl. Sci. 2020, 10, 2137. [CrossRef]

15. Lee, S.M.; Park, E.K.; Kim, I.Y.; Kim, S.I. An estimating method for systolic blood pressure by using pulse transit time and physical characteristic parameters. J. Electron. Eng. of Korea Syst. Control 2005, 42, 41-46.

16. Zhang, Q.; Zeng, X.; Hu, W.; Zhou, D. A Machine Learning-Empowered System for Long-Term Motion-Tolerant Wearable Monitoring of Blood Pressure and Heart Rate With Ear-ECG/PPG. IEEE Access 2017, 5, 10547-10561. [CrossRef]

17. Zheng, D.; Zhang, Y. A Ring-type Device for the Noninvasive Measurement of Arterial Blood Pressure. In Proceedings of the 25th Annual International Conference of the IEEE Engineering in Medicine and Biology Society (IEEE Cat. No.03CH37439), Cancun, Mexico, 17-21 September 2003; Institute of Electrical and Electronics Engineers (IEEE): New York City, NJ, USA, 2004; Volume 4, pp. 3184-3187.

18. Kim, S.C.; Cho, S.H. Analysis of the convergence algorithm model for estimating systolic and diastolic blood pressure based on two photoplethysmography. J. Korea Convergence Soc. 2019, 10, 53-58. [CrossRef]

19. Elgendi, M. On the analysis of fingertip photoplethysmogram signals. Curr. Cardiol. Rev. 2012, 8, 14-25. [CrossRef]

20. Pan, J.; Tompkins, W.J. A real-time QRS detection algorithm. IEEE Trans. Biomed. Eng. 1985, 32, $230-236$. [CrossRef] [PubMed]

21. Foo, J.Y.A.; Lim, C.S.; Wang, P. Evaluation of blood pressure changes using vascular transit time. Physiol. Meas. 2006, 27, 685-694. [CrossRef] [PubMed]

22. Bagha, S.; Shaw, L. A real time analysis of PPG signal for measurement of SpO2 and pulse rate. Int. J. Comput. Appl. 2011, 36, 45-50.

23. Henry, J.B.; Miller, M.C.; Kelly, K.C.; Champney, D. Mean arterial pressure (MAP): An alternative and preferable measurement to systolic blood pressure (SBP) in patients for hypotension detection during hemapheresis. J. Clin. Apher. 2002, 17, 55-64. [CrossRef] [PubMed] 
24. Atlasz, T.; Kellényi, L.; Kovacs, P.; Babai, N.; Thuroczy, G.; Hejjel, L.; Hernadi, I. The application of surface plethysmography for heart rate variability analysis after GSM radiofrequency exposure. J. Biochem. Biophys. Methods 2006, 69, 233-236. [CrossRef] [PubMed]

25. Alpert, B.S. Validation of the Welch Allyn Spot Vital Signs blood pressure device according to the ANSI/AAMI SP10: 2002. Accuracy and cost-efficiency successfully combined. Blood Press. Monit. 2007, 12, 345-347. [CrossRef] [PubMed]

26. Naschitz, J.E.; Itzhak, R.; Shaviv, N.; Khorshidi, I.; Sundick, S.; Isseroff, H.; Fields, M.; Priselac, R.M.; Yeshurun, D.; Sabo, E. Assessment of cardiovascular reactivity by fractal and recurrence quantification analysis of heart rate and pulse transit time. J. Hum. Hypertens. 2003, 17, 111-118. [CrossRef]

27. Chen, W.; Kobayashi, T.; Ichikawa, S.; Takeuchi, Y.; Togawa, T. Continuous estimation of systolic blood pressure using the pulse arrival time and intermittent calibration. Med Boil. Eng. 2000, 38, 569-574. [CrossRef] [PubMed]

28. Naschitz, J.E.; Bezobchuk, S.; Mussafia-Priselac, R.; Sundick, S.; Dreyfuss, D.; Khorshidi, I.; Karidis, A.; Manor, H.; Nagar, M.; Peck, E.R.; et al. Pulse transit time by R-wave-gated infrared photoplethysmography: Review of the literature and personal experience. J. Clin. Monit. 2004, 18, 333-342. [CrossRef]

29. Barschdorff, D.; Zhang, W.; Zhang, W.; D., B. Respiratory Rhythm Detection with Photoplethysmographic Methods. In Proceedings of the 16th Annual International Conference of the IEEE Engineering in Medicine and Biology Society, Baltimore, MD, USA, 3-6 November 1994; Institute of Electrical and Electronics Engineers (IEEE): New York City, NJ, USA. [CrossRef]

30. Marzorati, D.; Bovio, D.; Salito, C.; Mainardi, L.; Cerveri, P. Chest wearable apparatus for cuffless con-tinuous blood pressure measurements based on PPG and PCG signals. IEEE Access 2020, 8, 55424-55437. [CrossRef]

31. Ko, J.I.; Jeong, I.C.; Lee, D.H.; Park, S.W.; Hwang, S.O.; Park, S.M.; Kim, G.Y.; Joo, H.S.; Yoon, H.R. Com-pensation of error in noninvasive blood pressure measurement system using optical sensor. J. Biomed. Eng. Res. 2007, 28, 178-186.

32. Wentland, A.; Grist, T.M.; Wieben, O. Review of MRI-based measurements of pulse wave velocity: A biomarker of arterial stiffness. Cardiovasc. Diagn. Ther. 2014, 4, 193-206.

(C) 2020 by the authors. Licensee MDPI, Basel, Switzerland. This article is an open access article distributed under the terms and conditions of the Creative Commons Attribution (CC BY) license (http://creativecommons.org/licenses/by/4.0/). 